Department of Anatomy, Medical Theoretical Center, Technische Universität Dresden, Fetscherstrasse 74, 01307 Dresden, Germany

CRTD/DFG-Center for Regenerative Therapies Dresden-Cluster of Excellence, Tatzberg 47/49, 01307 Dresden, Germany

\section{Min Zhao}

Department of Dermatology and Department of Ophthalmology, Institute for Regenerative Cures, School of Medicine, University of California at Davis, Davis, CA 95817, USA

\# These authors contributed equally to this work.

\section{Abstract}

Endogenous electric fields (EF) may provide an overriding cue for directional cell migration during wound closure. Perceiving a constant direction requires active sodium-hydrogen exchanger (pNHE3) at the leading edge of HEK 293 cells but its activation mechanism is not yet fully understood. Because protein kinase $\mathrm{C}$ (PKC) is required in electrotaxis, we asked whether NHE3 is activated by PKC during wound healing. Using pharmacological (pseudosubstrate and edelfosine) inhibition, we showed that inhibition of $\mathrm{PKC} \eta$ isoform impairs directional cell migration in HEK 293 cells in the presence of a persistent directional cue $(0.25-0.3 \mathrm{~V} / \mathrm{mm}$ of EF for $2 \mathrm{~h}$ ). Further, we found that pNHE3 forms complexes with both PKC $\eta$ and $\gamma$-tubulin, suggesting that these molecules may regulate the microtubule-organizing center. In addition, cellular pNHE3 content was reduced significantly when PKC $\eta$ was inhibited during directional

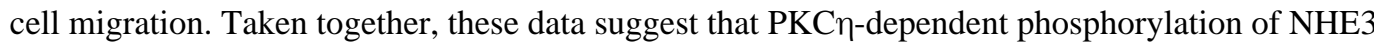
and the formation of $\mathrm{pNHE} 3 / \mathrm{PKC} \eta / \mathrm{\gamma}$-tubulin complexes at the leading edge of the cell are required for directional cell migration in an $\mathrm{EF}$.

\section{Keywords}

NHE3; PKC $\eta$; Resting membrane potential; Wound healing; Electric fields

\section{Introduction}

Successful wound healing relies heavily on effective cell migration with well-defined orientation and directionality at the wound edge. EFs of physiological strength $(\sim 1 \mathrm{~V} / \mathrm{cm})$, both endogenous and externally applied, are known to affect intracellular ion dynamics and induce dramatic changes in cell behavior, including cell directional motility [1-5]. Congruent with the overriding role of electric fields in wound healing [6], ion transport proteins have also been shown to modulate directional cell migration [7-11]. In the presence of a persistent cue of applied EFs, constant directionality was impaired when NHE3 was silenced in cells of osteogenic origin. Cells lost their characteristic polarity and orientation when NHE3 expression was blocked [12], suggesting a dependence of NHE3 activity on cell membrane shape [13]. Here, phospho- $\mathrm{Na}^{+} / \mathrm{H}^{+}$exchanger iso-form 3 (pNHE3) represents an interesting checkpoint by simultaneously having a direct modulation effect on cell membrane potential, an intrinsic physiological role (proton extrusion), and a biomechanical 
function for sensing EF and maintaining a constant cell direction [7, 12, 14, 15]. The mechanism of NHE3 activity in this novel area of cell guidance remains unknown.

PKC is involved in broad cellular cascades [16-20] including cell polarity and directional cell migration in general [21-24] and in electrotaxis [25]. Three distinct PKC iso-forms, $\theta$, $\eta$, and $\varepsilon$, are recruited by diacylglycerol (DAG) to microtubule-organizing center (MTOC), suggesting a direct implication of PKC in cell polarization [26]. MTOC reorientation depends on nuclear movement and the Par/PKC pathway is involved in keeping the MTOC immobile with respect to the moving nucleus [27]. PKC therefore represents a promising candidate as a multi-functional regulator of directional cell migration. Consistent with this existing evidence, our present study demonstrates that (1) the PKC $\eta$ isoform is implicated in maintaining directional cell migration because its absence resulted in a significantly delayed wound-healing rate and hyperpolarization of the cell membrane, (2) $\mathrm{PKC} \eta$ is responsible for NHE3 phosphorylation in cells migrating with a constant direction suggested by the very high colocalization rate between NHE3 and PKC $\eta$, and reduced pNHE3 content due to PKC $\eta$ inhibition in presence of constant directional cue, and (3) NHE3 phosphorylation via $\mathrm{PKC} \eta$ can induce appropriate cell polarity via recruitment to MTOC (pNHE3/PKC $\eta$ and $\mathrm{pNHE} 3 / \mathrm{\gamma}$-tubulin complex formation) in the presence of a constant directional cue.

\section{Materials and methods}

Direct-current electric field stimulation of cells, quantitative immunocytochemistry, fluorescence-activated cell sorter (FACS), real-time microscopy, cell motility assays, and in vivo wound-healing assay were performed as described previously [7, 28].

\section{Preparation of HEK cells}

We obtained 293T cells from the American Type Culture Collection (ATCC). Cells were cultured in Dulbecco's modified Eagle medium (DMEM)-Glutamax (Gibco, Germany) supplemented with $10 \%$ fetal calf serum (Biochrom, Germany). Cultures were maintained at $37{ }^{\circ} \mathrm{C}$ in humidified air and $5 \% \mathrm{CO}_{2}$. The cells were sub-cultured every third day for routine cell cultures.

A day before the experiment, cells were rinsed briefly with PBS (Biochrom, Germany) to remove all traces of serum that may contain trypsin inhibitors. Then, $500 \mu \mathrm{l}$ of $0.25 / 0.02 \%$ (w/v) of trypsin in EDTA (Biochrom, Germany) solution was added until the cells were dispersed (usually 1-2 min). Trypsin activity was then neutralized by adding $8.0-10.0 \mathrm{ml}$ of complete growth medium and the cell suspension transferred into a centrifugation tube by gentle pipetting. Cell count was determined by using a cell counter (CASY, Germany).

For FACS and Co-IP analyses, inocula of $1 \times 10^{6}$ viable cells $/ \mathrm{ml}$ were seeded on either side of a fibronectin $(10 \mu \mathrm{g} / \mathrm{ml})$ coated channel on an ibiTreat $\mu$-Slide I (Ibidi, Germany) and left undisturbed at $37{ }^{\circ} \mathrm{C}, 5 \% \mathrm{CO}_{2}$ for $1 \mathrm{~h}$. They are then supplemented with growth medium until the next day, during which the cells adhere and spread on the surface. 
For cell motility assays, immunofluorescence, and transfection studies, inocula of $5 \times 10^{5}, 5$ $\times 10^{4}$, or $8 \times 10^{4}$ viable cells $/ \mathrm{ml}$, respectively, were seeded onto the ibiTreat $\mu$-Slide I as above.

\section{Cell migration assay}

Time-lapse imaging was conducted using MetaMorph software and a Zeiss Axiovert 200 microscope with a 40× Fluor lens, as described previously [29]. ImageJ software was used to track cell migration and to quantify the migration distance, speed, and directedness. Cell migration tracking was performed manually by using a manual tracking plug-in for ImageJ along with the Chemotaxis and Migration tool functions (Ibidi, Germany). Data analysis was conducted as previously described $[28,29]$. Cell migration trajectories were accumulated and superimposed on individual plots, with the cell origin (position at start of experiment, time zero) placed in the center $(0,0)$ (see Fig. 2). Black lines represent cells migrating toward the anode (left), and red lines represent cells migrating toward the cathode (right).

\section{PKC-knockout mouse}

$\mathrm{PKC}^{-/-}, \mathrm{PKC}^{-/-}$, and $\mathrm{PKC} \eta \theta^{-/-} \mathrm{C} 57 \mathrm{BL} 6$ mice were generated in the Gascoigne lab (The Scripps Research Institute, La Jolla, CA, USA), as previously described [30].

\section{Wound-healing assay}

Six age-matched mice ( 8 weeks old) from each group were used in the wound-healing assay. The animals were anesthetized with intramuscular injection of ketamine $100 \mathrm{mg} / \mathrm{kg}$ with acepromazine $2.5 \mathrm{mg} / \mathrm{kg}$. A circular trephine of $\varphi 3 \mathrm{~mm}$ diameter was used to mark the center of the left cornea of each mouse under the dissecting microscope, and a circular epithelial wound made by carefully scraping off the epithelial layer up to the trephine marking boundary. Artificial tear solution $(122.18 \mathrm{mM} \mathrm{NaCl}, 5.1 \mathrm{mM} \mathrm{KCl}, 1.05 \mathrm{mM}$ $\mathrm{CaCl}_{2} \cdot 2 \mathrm{H}_{2} \mathrm{O}, 0.98 \mathrm{mM} \mathrm{MgCl}_{2}, 2.96 \mathrm{mM} \mathrm{Na}_{2} \mathrm{HPO}_{4}, 25 \mathrm{mM} \mathrm{NaHCO}_{3}, 5.11 \mathrm{mM}$ D-glucose, $0.3 \mathrm{mM}$ glutathione disulfide, $\mathrm{pH}$ 6.85) was used to moisturize the cornea surface during the operation. Clinical fluorescein dye was used to stain the wound area, and photographed with a digital camera at $0,12,24,36$, and $48 \mathrm{~h}$ post-operation. Mice were anesthetized at each time point as described above. Wound healing was analyzed by measuring the remaining wound areas using ImageJ software.

\section{Inhibitors and antibodies}

When applicable, (i) the phosphatidylinositol-specific phospholipase c (PI-PLC) inhibitor edelfosine (Tocris Biosciences, Germany), (ii) the PKC $\eta$ inhibitor pseudosubstrate (USBiological, USA), (iii) PKC inhibitor, GÖ 6983 (Biomol International, Germany), or (iv) NHE3 inhibitor S3226 (Sanofi Aventis, Germany) were added to either the media or to the HBSS. The drug concentrations were set as $20 \mu \mathrm{M}$ (edelfosine); $15 \mu \mathrm{M}$ (pseudosubstrate); $0.5 \mu \mathrm{M}$ (GÖ 6983); $10 \mu \mathrm{M}$ (S3226) except where otherwise stated.

Cells were incubated with primary antibody [mouse monoclonal anti-phospho NHE-3 (Ser 552; 1:1,000), rabbit polyclonal PKC $\eta$ (1:300), mouse monoclonal anti-gamma tubulin (Sigma Aldrich, Germany; 1:4,000), rabbit polyclonal PKC $\eta$ (1:500), rabbit polyclonal antigamma tubulin (Abcam, Germany; 1:250), or mouse monoclonal anti-phospho NHE-3 (Ser 
552; 1: 1,000)] overnight at $4{ }^{\circ} \mathrm{C}$. Cells were washed three times with phosphate-buffered saline (PBS) and then incubated with secondary antibody [Texas Red goat anti-mouse (1:1,000), FITC goat anti-rabbit (1:500), Texas Red goat anti-mouse (1:800), FITC goat anti-rabbit (1:800), FITC Goat anti-rabbit $(1: 1,000)$, or Texas Red goat anti-mouse (1:1,000); Jackson ImmunoRe-search Laboratories, USA] for $1 \mathrm{~h}$ at room temperature (RT) in darkness. Finally, cells were rinsed in PBS.

\section{Co-immunoprecipitation}

Prior to lysis, cells were stimulated with applied EF for $5 \mathrm{~h}(0.3 \mathrm{~V} / \mathrm{mm})$ in the absence (control) or presence of inhibitor S3226. After stimulation, cells were washed gently with PBS, trypsinized to detach them from the $\mu$ slides, and centrifuged. The cell pellet was then resuspended in RIPA buffer supplemented with complete, mini, EDTA-free pro-tease cocktail inhibitor (Roche, Germany) and was given constant agitation for 30 min at $4{ }^{\circ} \mathrm{C}$. Next the cells were centrifuged at $5,000 \mathrm{rpm}$ for $15 \mathrm{~min}$ at $4{ }^{\circ} \mathrm{C}$. The super-natant was transferred into new vials and the extracts were clarified by incubation with Pierce protein A/G Agarose beads (Thermo Scientific, Germany) for $30 \mathrm{~min}$ at $4{ }^{\circ} \mathrm{C}$ with constant agitation and followed by a brief centrifugation for $5 \mathrm{~min}$ at $600 \times \mathrm{g}$. To pull down the pNHE3-PKC $\eta$ or pNHE3- $\gamma$ tubulin complexes, the pre-clear lysates were incubated with either rabbit polyclonal PKC $\eta$ (1:20, Santa Cruz Biotechnology, Germany), or mouse monoclonal antiphospho NHE-3 (Ser 552, 1:200, Novus Biologicals, Germany), respectively, overnight at 4 ${ }^{\circ} \mathrm{C}$. The resulted immuno-complexes were collected by further incubation with immobilized protein $\mathrm{A} / \mathrm{G}$ beads for $3 \mathrm{~h}$ at $4{ }^{\circ} \mathrm{C}$ with constant agitation. The immunoprecipitated proteins with the beads were washed three times with RIPA lysis buffer (centrifuged each time at $5,000 \mathrm{rpm}$ for $5 \mathrm{~min}$ at $4{ }^{\circ} \mathrm{C}$ ). Precipitated proteins were then eluted from the beads via the addition of 6 loading buffer to the lysate and incubated for $5 \mathrm{~min}$ at $95 \times{ }^{\circ} \mathrm{C}$. Consequently, the samples were run on SDS-PAGE. For negative controls, pre-clear lysates were incubated without primary antibody to show the absence of its interacting protein. (Before the use of beads, the beads were washed thrice with RIPA buffer and centrifuged at 5,000 rpm for 5 min each time).

\section{Western blot}

The immunoprecipitates were heated at $95{ }^{\circ} \mathrm{C}$ for $5 \mathrm{~min}$. Proteins from the cell lysates were separated based on their size by an SDS gel that contained $10 \%$ polyacrylamide (Roth, Germany) and then transferred to WestClear nitro-cellulose membranes (GenScript, USA) of $0.2-\mu \mathrm{m}$ pore size. The process after the protein transfer to the membrane was performed a using ONE-HOUR Western Basic Kit (GenScript, USA). During the protein transfer to the membranes, "mixture 1" was prepared by mixing the primary antibody to the WB-1 solution at a ratio of $1 \mu \mathrm{g}$ in $10 \mu \mathrm{l}$ (mouse monoclonal anti-phospho NHE-3 (Ser 552), rabbit polyclonal $\mathrm{PKC} \eta$ ). After the gel transfer the membranes were pretreated with the pretreatment solution (prepared by mixing pretreat solution A with pretreat solution B at 1:1 ratio) and incubated on a shaker at RT for $5 \mathrm{~min}$. The membranes were then rinsed twice with $1 \times$ wash solution. Finally, the blots were incubated with WB-2 solution containing mixture 1 on a shaker at RT for $40 \mathrm{~min}$. The membranes were then rinsed and washed thrice for 10 min each on the shaker with $1 \times$ wash solution. Blots were developed with Lumisensor Chemiluminescent HRP substrate using image reader-LAS 3,000 (Fujifilm, Germany). 


\section{Statistics}

Statistical calculations were performed using paired or unpaired Student's $t$ tests, or ANOVA. A $p<0.05(*), p<0.01(* *)$ or $p<0.001(* * *)$ was defined as significant. Data are presented as mean \pm SEM.

\section{Results}

\section{Wound-healing rate decreased significantly in PKC $\eta$ knockout mouse cornea}

A circular cornea wound was generated in vivo on wild-type and PKC knockout mice (Fig. 1a). Wound areas were measured at $0 \mathrm{~h}$ and at $24 \mathrm{~h}$ in wild-type and PKC knockout (eta $-\eta-$-, theta $-\theta$-, and double $-\eta \theta-$ ) animals in situ to determine which isoform has a greater impact in wound healing (Fig. 1b). This revealed that wound-healing degree varied depending on the genetic deletion of different PKC isoforms. PKC $\eta$ knockout mouse cornea showed the most delayed healing $\left(0.5 \mathrm{~mm}^{2}\right)$ at $24 \mathrm{~h}$ after wounding, compared to the wild-type control $\left(0.1 \mathrm{~mm}^{2}\right)$ and PKC $\theta$ knockout $\left(0.1 \mathrm{~mm}^{2}\right)$. PKC $\theta \eta$ double knockout showed significantly delayed wound healing $\left(0.7 \mathrm{~mm}^{2}\right)$.

\section{Inhibition of PKC $\eta$ impaired directional cell motility}

In the presence of specific inhibitors against PI-PLC, PKC $\eta$, and PKC isoforms, the migratory parameters such as migration speed and the directedness of migration were affected. Cell migration speed was significantly increased by 3.1 -fold in the presence of

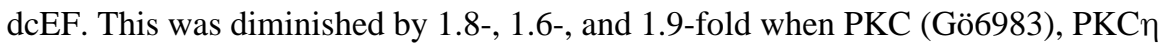
(pseudosubstrate) or PI-PLC (edelfosine) were inhibited (Fig. 2a).

The directedness values of migrating cells at different conditions [control (without EF), EF alone, or EF with inhibitors Gö6983, pseudosubstrate or edelfosine] was analyzed manually using the manual tracking plug-in for ImageJ along with the Chemotaxis and Migration tool software (Ibidi, Germany). The migration directedness was significantly decreased when PKC $\eta$ or PI-PLC was inhibited using pseudosubstrate or edelfosine, respectively (Fig. 2b). Gö6983, a nonspecific inhibitor of PKC isoforms, did not cause any dramatic change in the cell directedness. In the plots shown in Fig. 2c-g (and in Supplementary movies 1-5), each dot represents a cell and the line connecting the circle from the center of axis $(0,0)$ is the trajectory of the cell migration. In the absence of EF, cells migrated randomly (Fig. 2c). EF treatment triggered directional migration (electrotaxis) of the cells, with $100 \%$ of the cells showing cathodal migration, and the majority of the cells migrating more persistently along or close to the $\mathrm{X}$ axis with long migration trajectories (Fig. 2d). Nonspecific PKC inhibitor Gö6983 changed the electrotactic trajectory pattern: (1) A small proportion of anodal migrating cells was evident; (2) Cathodally migrating cells show more scattered trajectory away from the $\mathrm{X}$ axis, indicating less directedness along the $\mathrm{EF}$ vector; and (3)

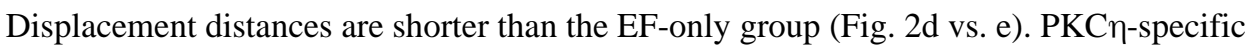
inhibitor (pseudosubstrate, Fig. 2f) and PI-PLC inhibition (Fig. 2g, edelfosine) further reduced the electrotactic response in comparison to EF only group, with: (1) More cells moving back and forward across the $\mathrm{Y}$ axis, switching migration direction between cathode and anode; (2) Displacement distances are much shorter than EF only group, and more distorted trajectories indicating hesitant directed migration. Time-lapse analyses further 
revealed that in contrast to the no drug control group, Gö6983, pseudosubstrate, and edelfosine all significantly reduced the electrotactic response throughout the entire time course (Fig. 2b).

pNHE3 formed complexes with PKC $\eta$ and $\gamma$-tubulin thereby associating with MTOC

To characterize the interaction of phosphorylated NHE3 with $\gamma$-tubulin and PKC $\eta$, coimmunoprecipitation assays were performed. Protein interactions in the cells were observed at different conditions such as no EF control, EF treated for $5 \mathrm{~h}(0.3 \mathrm{~V} / \mathrm{mm})$, and EF treatment in the presence of NHE3 inhibitor S3226. Bands corresponding to pNHE3 and $\gamma-$ tubulin were detected in the immunoprecipitated cell lysates pulled down with PKC (Fig. 3a) and pNHE3 (Fig. 3b) antibody, respectively. Negative control was performed on both immunoprecipitation and supernatant in the absence of PKC $\eta$ or pNHE3 antibodies for pNHE3/PKC $\eta$ (Supplementary Fig. 1a) or pNHE3/ $\gamma$-tubulin (Supplementary Fig. 1b) pull down, respectively. The pull-down assay suggests that interaction exists between pNHE3/ $\mathrm{PKC} \eta$ and $\mathrm{pNHE} 3 / \gamma$-tubulin (Fig. 3). Interestingly, $\mathrm{EF}$ triggered an up-regulation of pNHE3/PKC $\eta$ interaction with more co-immunoprecipitation compared with no EF control (Fig. 3a). In the presence of EF, pharmacological inhibition of NHE3 (S3226) impaired the interaction between pNHE3/PKC $\eta$ and pNHE3/ $\gamma$-tubulin (Fig. 3), suggesting that physiological activity of NHE3 is required for the EF-promoted interaction between the proteins. This was confirmed with quantification of protein levels as a percentage (\%) value in cell lysate versus immunoprecipitates (Fig. 3c). These findings are also consistent with the immunofluorescence studies showing that pNHE3 colocalizes with PKC $\eta$ and with $\gamma$ tubulin during directional cell migration.

\section{pNHE3 colocalized with PKC $\eta$ and $\gamma$-tubulin at filopodia and MTOC}

It is known that pNHE3 associates with the cytoskeletal protein $\beta$ actin at the leading edge [7]. Using immunofluorescence, we found that pNHE3 colocalizes with $\mathrm{PKC} \eta$ at membrane protrusions of polarized cells and also with $\gamma$-tubulin at the MTOC. Control cells showed bulky patch-like accumulation of NHE3 all through the cell, which are less mobile when compared to the cells in the electric field. The localization of these proteins fills almost the entire area of the cell except for the nuclear region. In the presence of EF, cells show a distinct pattern of polarization with a well-defined cell front and back. Clear, distinct patchlike accumulations of NHE3 and PKC $\eta$ at the leading edge and at cell boundaries were detectable. Interestingly, the accumulations tended to move slightly away from the leading edge upon EF application, however it is not clear what causes this translocation of proteins backwards after attaining polarization. Significant colocalization occurred at both filopodia and MTOC in polarized cells (Fig. 4a-c and Supplementary movies 6-9). To quantify the efficiency of colocalization, a Pearson coefficiency assay was conducted to quantify the overlapping fluorescence pixels at filopodia and MTOC regions. The colocalization efficiency between pNHE3 and PKC $\eta$ was increased by $50 \pm 0.02 \%$ at filopodia and by 17 $\pm 0.01 \%$ at MTOC (Fig. $4 \mathrm{~d}$ ). PKC $\eta$ and $\gamma$-tubulin had the same values as pNHE3 and PKC $\eta$ (Fig. 4e). Between pNHE3 and $\gamma$-tubulin, the increase was $25 \pm 0.02$ and $15.6 \pm 0.01 \%$ at filopodia and MTOC, respectively (Fig. 4f). When cells were treated with $5 \mu \mathrm{M}$ of pseudosubstrate or edelfosine, their polarity was dramatically disturbed and the accumulations in these cells showed the pattern similar to control cells. The cell boundaries 
could not be clearly demarcated when compared with the EF-treated cells (Supplementary

Fig. 2).

\section{PKC $\eta$ activated NHE3 via a PI-PLC-independent mechanism}

We previously showed that NHE3 is activated via a PI3K/Akt-independent pathway during persistent directional cell migration. This led us to investigate whether PKC, especially the eta isoform, might be responsible for NHE3 phosphorylation. Cells were exposed to specific inhibitors and analyzed by flow cytometry. Pseudosubstrate and edelfosine resulted in significant inhibition of PKC $\eta$ and PI-PLC, respectively. Cells exposed to EF showed a significant increase in production of both pNHE3 and PKC $\eta$ compared to the control cells (Fig. 5a, d). Pseudo-substrate, a PKC $\eta$-specific inhibitor, caused a decrease in the intracellular levels of pNHE3 (Fig. 5a, c). When cells were treated with edelfosine, a PIPLC-inhibitor, pNHE3 level increased (Fig. 5a, b). Edelfosine exposure caused an increase in PKC $\eta$ level (Fig. 5d, e) while pseudosubstrate significantly decreased the intracellular level of PKC $\eta$ (Fig. 5d, f). These results suggest that PKC $\eta$ is not activated by PI-PLC.

\section{PKC $\eta$ could change $V_{\text {mem }}$ during electrotaxis}

Upon electric field stimulation, cells migrate in a defined direction, possibly due to changes in cell morphology. This causes a change of their volume, which influences the membrane potential. The fluorescence of Bis-(1,3-Dibutylbarbituric Acid)Trimethine Oxonol $\left(\mathrm{DiBAC}_{4}(3)\right)$ dye increases as a result of membrane depolarization. In the presence of pseudosubstrate or edelfosine cells had relatively hyperpolarized cell membrane, 36.8 and $15.2 \%$, respectively, compared to EF treatment alone (Fig. 6a). However, pseudosubstrate caused a bigger shift in $V_{\text {mem }}$ (Fig. 6b) than edelfosine (Fig. 6c). This may suggest that $\mathrm{PKC} \eta$ is required for EF-induced depolarization of migrating cells. Gramicidin, used as a depolarization control, resulted in a significant shift in $\mathrm{DiBAC}_{4}(3)$ fluorescence intensity.

\section{Discussion}

A functional link between NHEs and cell polarity has been previously shown [7, 31, 32]. As EFs were used as a cue for studying the changes in the cells [1,2, 6, 33, 34], electric fields of $0.3 \mathrm{~V} / \mathrm{mm}$ were applied, which mediated the motility causing an increase of speed along with the cathodal directionality in the 293T cell type. Moreover, increases in $V_{\text {mem }}$ suggest that the endogenous EF acts as a sensor $[1,6]$ and decreased membrane potential in the $\mathrm{PKC} \eta$ inhibited cells indicate the role of $\mathrm{PKC} \eta$ isoform in modulating the ion conductance $[35,36]$. The decreased protein expression levels of pNHE3 when PKC $\eta$ was inhibited explain the dependency of NHE3 phosphorylation on PKC $\eta$.

\section{Cellular directedness is regulated by the activity of PKC during electrotaxis}

Impaired cell polarity leads to increased cell spreading and reduces cell motility [37]. EFinduced cells showed greater directedness than those of control cells $[1,6]$. Inhibitory studies significantly decreased the motility with reduced directionality. Tracking of individual cells showed EF-directed cathodal migration with an increased motility. An intriguing possibility is that the trailing edge of the cell may drive cell migration by forming a defined rear prior to the formation of a polarized cell protrusion [38]. EF-induced cell 
directedness was attenuated by $42.5 \%$ when PKC $\eta$ was inhibited by the use of specific inhibitor PKC $\eta$ pseudosubstrate. These results are consistent with studies showing that disruption of protein kinase $\mathrm{C} \eta$ results in impairment of wound healing [39]. Similarly, when cells were inhibited by PI-PLC and PKC inhibitors, cell directedness was disturbed by 25 and $15 \%$, respectively. Even in the presence of a persistent EF cue, the speed of migrating cells in the presence of PKC $\eta$ and PI-PLC inhibitors was decreased by 9.7- and 16.8-fold, respectively, when compared to control cells, indicating the role of $\mathrm{PKC} \eta$ during directional migration. This supports that the existence of PKC isoforms within the cell can be important in regulating lipid-dependent signal transduction [40].

\section{PKC $\eta$ associates with pNHE3 and $\gamma$ tubulin to promote the cell polarity during migration}

Previous studies showed the interaction between cytoskeleton proteins and ion transporters during cell migration [12, 41, 42]. Besides regulating ion fluxes, ion transporters are also involved in interactions with membrane proteins. Inhibition of $\mathrm{PKC} \eta$ does not perturb establishment of the front rear axis, but affects its orientation and induces random cell migration to a certain extent (Supplementary Fig. 2 and movie 4). Regulation or colocalization of pNHE3-PKC $\eta$ may promote persistent and directed cell migration by stabilizing the microtubule network. Our results show a strong correlation between these complexes at filopodia and cytoplasmic regions in a directionally migrating cell.

Similarly, good correlation is observed between PKC $\eta$ and $\gamma$-tubulin at filopodia and MTOC regions in a polarized cell. This is also congruent with studies showing that $\mathrm{PKC} \eta$ gets recruited at the MTOC for polarization [26]. Localization of NHE3 with $\gamma$ tubulin at the MTOC was confirmed by co-immunoprecipitation studies. In the migrating cells, PKCdependent NHE3 activation might lead to phosphorylation of NHE3, which is in turn responsible for clustering or accumulation of pNHE3/PKC $\eta$ complexes at microtubule "plus" ends, i.e., leading edges and also at the MTOC.

\section{PKC induced changes in the membrane potential $\left(V_{\text {mem }}\right)$ during directional cell migration}

To obtain further insights into the role of PKC-dependent NHE3 activity during directed cell migration, FACS analysis was performed to observe membrane potential variances in the protein expression levels. Exposing cells to an EF causes changes in plasma membrane potentials [43]. These changes involve different membrane proteins, such as ion channels, transporters, and receptors, and may also involve $\mathrm{Ca}^{2+}$ signaling $[5,44,45]$. Depolarization of cells with Gramicidin results in a large influx of $\mathrm{Ca}^{2+}$ ions, which results in a calcium wave that may be another source for increase in the DAG levels on Golgi [46] and which might also activate PKC. In the presence of PKC $\eta$ and PIPLC inhibitors, cell membrane was hyperpolarized by 38 and $15 \%$, respectively, compared to EF stimulated cells. Cells with altered PI-PLC or PKC $\eta$ activity migrated but exhibited a significantly reduced ability to move directionally in response to electric cues. Membrane hyperpolarization inhibits $\mathrm{Ca}^{2+}$ signaling and thereby affects electrotaxis [43]. Electric potential gradients may induce polarized signaling pathways through activation of membrane receptors on the cathodal facing side, then the downstream intra-cellular signaling pathways, which culminate in polarized actin polymerization and directional cell migration [29, 47, 48]. 


\section{Involvement of PKC-dependent NHE3 activity}

The intracellular levels of PKC $\eta$ compared to pNHE3 were measured by FACS analysis. In order for PKC isozymes to phosphorylate NHE3, PKC has to be initially activated. As the PLC pathway is one of the routes through which PKCs are activated by DAG [49-51], cells were inhibited with PIPLC. Interestingly, in the presence of inhibitor, there was an increase in the levels of $\mathrm{PKC} \eta$ and pNHE3, suggesting that the PKC $\eta$ may take over another route for its activation (Fig. 7). When PKC $\eta$ is inhibited to test how it affects the levels of pNHE3, the results showed a decrease in levels of pNHE3, suggesting that NHE3 activity at the leading edge during directional cell migration depends on PKC.

As the phosphorylation of NHE3 plays a role in its sub-cellular trafficking in vivo [52], the interaction PKC $\eta$ with pNHE3 was confirmed by co-immunoprecipitation studies. As negative controls, pre-clear lysates without primary antibody showed the absence of its interacting protein. The interaction and colocalization of PKC $\eta$ with $\gamma$-tubulin was confirmed, which is consistent with studies showing that $\mathrm{PKC} \eta$ is recruited at the MTOC for its reorientation and polarization [26]. The localization of pNHE3 at the MTOC, and its interaction with $\gamma$-tubulin, was confirmed by immunofluorescence and immunoprecipitation studies, respectively.

Live cell imaging of NHE3 and PKC -transfected cells showed NHE3 in the form of patchlike accumulations, which are less mobile in the randomly migrating cells than in EFinduced cells. Upon stimulation of cells with EF, there is relocation of these patches away from the leading edge and the cells become highly polarized. Both the polarity and distribution of the proteins is disturbed when the cells were inhibited with drugs, and cells also showed certain morphological characteristics similar to the randomly migrating cells.

Taken together, our data suggest that PKC $\eta$-dependent NHE3 activity, probably activated by intracellular $\mathrm{Ca}^{2+}$, is required for polarization and orientation in directionally migrating cells and may play an important role in wound healing.

\section{Supplementary Material}

Refer to Web version on PubMed Central for supplementary material.

\section{Acknowledgments}

We thank K. Pehlke for her skillful assistance. We thank R. Gao and K. Nakajima for their help during cell motility assays and S. Perike for his help in FACS and biochemical assays. We also thank N. Gascoigne for providing PKC knockout mice. This work was supported by the German Research Foundation (DFG Research Fellowship to NÖ, OE541/1-1) and by MeDDrive-Grant from the Medical Faculty of Technical University of Dresden (Özkucur_60.246). BS was supported by the Royal Society URF award UF051616, and the European Research Council StG grant 243261. Work in the laboratory of MZ was also supported by National Science Foundation Grant MCB-0951199, California Institute of Regenerative Medicine Research Grant RB1-01417 (to MZ), and in part by an Unrestricted Grant from Research to Prevent Blindness to University of California, Davis, Ophthalmology. This work was also supported by NIH-NEI R01-EY-019101.

\section{References}

1. Levin M. Large-scale biophysics: ion flows and regeneration. Trends Cell Biol. 2007; 17:261-270. [PubMed: 17498955] 
2. Tai G, Reid B, Cao L, Zhao M. Electrotaxis and wound healing: experimental methods to study electric fields as a directional signal for cell migration. Methods Mol Biol. 2009; 571:77-973. [PubMed: 19763960]

3. Forrester JV, Lois N, Zhao M, McCaig C. The spark of life: the role of electric fields in regulating cell behaviour using the eye as a model system. Ophthalmic Res. 2007; 39:4-16. [PubMed: 17164572]

4. Funk RH, Monsees T, Ozkucur N. Electromagnetic effects: from cell biology to medicine. Prog Histochem Cytochem. 2009; 43:177-264. [PubMed: 19167986]

5. Ozkucur N, Epperlein HH, Funk RH. Ion imaging during axolotl tail regeneration in vivo. Dev Dyn. 2010; 239:2048-2057. [PubMed: 20549718]

6. Zhao M. Electrical fields in wound healing: an overriding signal that directs cell migration. Semin Cell Dev Biol. 2009; 20:674-682. [PubMed: 19146969]

7. Ozkucur N, Perike S, Sharma P, Funk RH. Persistent directional cell migration requires ion transport proteins as direction sensors and membrane potential differences in order to maintain directedness. BMC Cell Biol. 2011; 12:4. [PubMed: 21255452]

8. Ozkucur N, Monsees TK, Perike S, Do HQ, et al. Local calcium elevation and cell elongation initiate guided motility in electrically stimulated osteoblast-like cells. PLoS One. 2009; 4:e6131. [PubMed: 19584927]

9. Schwab A, Wulf A, Schulz C, Kessler W, et al. Subcellular distribution of calcium-sensitive potassium channels (IK1) in migrating cells. J Cell Physiol. 2006; 206:86-94. [PubMed: 15965951]

10. Djamgoz MBA, Mycielska M, Madeja Z, Fraser SP, et al. Directional movement of rat prostate cancer cells in direct-current electric field: involvement of voltage-gated $\mathrm{Na}^{+}$channel activity. $\mathrm{J}$ Cell Sci. 2001; 114:2697-2705. [PubMed: 11683396]

11. Wei C, Wang X, Chen M, Ouyang K, et al. Calcium flickers steer cell migration. Nature. 2009; 457:901-905. [PubMed: 19118385]

12. Perike S, Ozkucur N, Sharma P, Staroske W, et al. Phospho-NHE3 forms membrane patches and interacts with beta-actin to sense and maintain constant direction during cell migration. Exp Cell Res. 2014; 324:13-29. [PubMed: 24657527]

13. Alexander RT, Malevanets A, Durkan AM, Kocinsky HS, et al. Membrane curvature alters the activation kinetics of the epithelial $\mathrm{Na}^{+} / \mathrm{H}^{+}$exchanger, NHE3. J Biol Chem. 2007; 282:73767384. [PubMed: 17218318]

14. Bunnell TM, Burbach BJ, Shimizu Y, Ervasti JM. Beta-actin specifically controls cell growth, migration, and the G-actin pool. Mol Biol Cell. 2011; 22:4047-4058. [PubMed: 21900491]

15. Alexander RT, Jaumouille V, Yeung T, Furuya W, et al. Membrane surface charge dictates the structure and function of the epithelial $\mathrm{Na}^{+} / \mathrm{H}^{+}$exchanger. EMBO J. 2011; 30:679-691. [PubMed: 21245831]

16. Vargas-Lopes C, Madeira C, Kahn SA, Albino do Couto I, et al. Protein kinase C activity regulates d-serine availability in the brain. J Neurochem. 2011; 116:281-290. [PubMed: 21070240]

17. Reyland ME. Protein kinase $\mathrm{C}$ isoforms: multi-functional regulators of cell life and death. Front Biosci. 2009; 14:2386-2399.

18. Battaini F, Pascale A. Protein kinase C signal transduction regulation in physiological and pathological aging. Ann N Y Acad Sci. 2005; 1057:177-192. [PubMed: 16399894]

19. Rivera R, Rozas JL, Lerma J. PKC-dependent autoregulation of membrane kainate receptors. EMBO J. 2007; 26:4359-4367. [PubMed: 17898803]

20. Kawasaki T, Ueyama T, Lange I, Feske S, et al. Protein kinase C-induced phosphorylation of Orai1 regulates the intracellular $\mathrm{Ca}^{2+}$ level via the store-operated $\mathrm{Ca}^{2+}$ channel. J Biol Chem. 2010; 285:25720-25730. [PubMed: 20534587]

21. Tuomi S, Mai A, Nevo J, Laine JO, et al. PKC epsilon regulation of an alpha5 integrin-ZO-1 complex controls lamellae formation in migrating cancer cells. Sci Signal. 2009; 2:ra32. [PubMed: 19567915]

22. Nishimura T, Kaibuchi K. Numb controls integrin endocytosis for directional cell migration with aPKC and PAR-3. Dev Cell. 2007; 13:15-28. [PubMed: 17609107]

23. Larsson C. Protein kinase $C$ and the regulation of the actin cytoskeleton. Cell Signal. 2006; 18:276-284. [PubMed: 16109477] 
24. Etienne-Manneville S, Hall A. Cell polarity: Par6, aPKC and cytoskeletal crosstalk. Curr Opin Cell Biol. 2003; 15:67-72. [PubMed: 12517706]

25. Nuccitelli R, Smart T, Ferguson J. Protein kinases are required for embryonic neural crest cell galvanotaxis. Cell Motil Cytoskelet. 1993; 24:54-66.

26. Quann EJ, Liu X, Altan-Bonnet G, Huse M. A cascade of protein kinase C isozymes promotes cytoskeletal polarization in T cells. Nat Immunol. 2011; 12:647-654. [PubMed: 21602810]

27. Gomes ER, Jani S, Gundersen GG. Nuclear movement regulated by Cdc42, MRCK, myosin, and actin flow establishes MTOC polarization in migrating cells. Cell. 2005; 121:451-463. [PubMed: 15882626]

28. Song B, Gu Y, Pu J, Reid B, et al. Application of direct current electric fields to cells and tissues in vitro and modulation of wound electric field in vivo. Nat Protoc. 2007; 2:1479-1489. [PubMed: 17545984]

29. Zhao M, Song B, Pu J, Wada T, et al. Electrical signals control wound healing through phosphatidylinositol-3-OH kinase-gamma and PTEN. Nature. 2006; 442:457-460. [PubMed: 16871217]

30. Fu G, Vallee S, Rybakin V, McGuire MV, et al. Themis controls thymocyte selection through regulation of T cell antigen receptor-mediated signaling. Nat Immunol. 2009; 10:848-856. [PubMed: 19597499]

31. Simons M, Gault WJ, Gotthardt D, Rohatgi R, et al. Electrochemical cues regulate assembly of the Frizzled/Dishevelled complex at the plasma membrane during planar epithelial polarization. Nat Cell Biol. 2009; 11:286-294. [PubMed: 19234454]

32. Frantz C, Karydis A, Nalbant P, Hahn KM, et al. Positive feedback between Cdc42 activity and $\mathrm{H}^{+}$ efflux by the Na-H exchanger NHE1 for polarity of migrating cells. J Cell Biol. 2007; 179:403410. [PubMed: 17984318]

33. McCaig CD, Rajnicek AM, Song B, Zhao M. Controlling cell behavior electrically: current views and future potential. Physiol Rev. 2005; 85:943-978. [PubMed: 15987799]

34. Levin M. Molecular bioelectricity in developmental biology: New tools and recent discoveries. Bioessays. 2012; 34:205-217. [PubMed: 22237730]

35. Moolenaar WH, Tertoolen LGJ, de Laat SW. Phorbol ester and diacylglycerol mimic growth factors in raising cytoplasmic pH. Nature. 1984; 312:371-374. [PubMed: 6095096]

36. Rosoff PM, Stein LF, Cantley LC. Phorbol esters induce differentiation in a pre-B-lymphocyte cell line by enhancing $\mathrm{Na}^{+} / \mathrm{H}^{+}$exchange. J Biol Chem. 1984; 259:7056-7060. [PubMed: 6144681]

37. Sander EE, ten Klooster JP, van Delft S, van der Kammen RA, et al. Rac downregulates rho activity. J Cell Biol. 1999; 147:1009-1022. [PubMed: 10579721]

38. Vicente-Manzanares M, Koach MA, Whitmore L, Lamers ML, et al. Segregation and activation of myosin IIB creates a rear in migrating cells. J Cell Biol. 2008; 183:543-554. [PubMed: 18955554]

39. Chida K, Hara T, Hirai T, Konishi C, et al. Disruption of protein kinase Ceta results in impairment of wound healing and enhancement of tumor formation in mouse skin carcinogenesis. Cancer Res. 2003; 63:2404-2408. [PubMed: 12750259]

40. Ron D, Kazanietz MG. New insights into the regulation of protein kinase C and novel phorbol ester receptors. FASEB J. 1999; 13:1658-1676. [PubMed: 10506570]

41. Yun CHC, Lamprecht G, Forster DV, Sidor A. NHE3 kinase A regulatory protein E3KARP binds the epithelial brush border $\mathrm{Na}^{+} / \mathrm{H}^{+}$exchanger NHE3 and the cytoskeletal protein ezrin. $\mathrm{J}$ Biol Chem. 1998; 273:25856-25863. [PubMed: 9748260]

42. Zhao H, Shiue H, Palkon S, Wang Y, et al. Ezrin regulates NHE3 translocation and activation after $\mathrm{Na}^{+}$-glucose cotransport. Proc Natl Acad Sci USA. 2004; 101:9485-9490. [PubMed: 15197272]

43. Gao RC, Zhang XD, Sun YH, Kamimura Y, et al. Different roles of membrane potentials in electrotaxis and chemotaxis of dictyostelium cells. Eukaryot Cell. 2011; 10:1251-1256. [PubMed: 21743003]

44. Dai S, Hall DD, Hell JW. Supramolecular assemblies and localized regulation of voltage-gated ion channels. Physiol Rev. 2009; 89:411-452. [PubMed: 19342611]

45. Shanley LJ, Walczysko P, Bain M, MacEwan DJ, et al. Influx of extracellular $\mathrm{Ca}^{2+}$ is necessary for electrotaxis in dictyostelium. J Cell Sci. 2006; 119:4741-4748. [PubMed: 17077123] 
46. Gallegos LL, Kunkel MT, Newton AC. Targeting protein kinase C activity reporter to discrete intracellular regions reveals spatiotemporal differences in agonist-dependent signaling. J Biol Chem. 2006; 281:30947-30956. [PubMed: 16901905]

47. Zhao M, Pu J, Forrester JV, McCaig CD. Membrane lipids, EGF receptors, and intracellular signals colocalize and are polarized in epithelial cells moving directionally in a physiological electric field. FASEB J. 2002; 16:857-859. [PubMed: 11967227]

48. Huttenlocher A, Horwitz AR. Wound healing with electric potential. N Engl J Med. 2007; 356:303-304. [PubMed: 17229960]

49. Bianco R, Melisi D, Ciardiello F, Tortora G. Key cancer cell signal transduction pathways as therapeutic targets. Eur J Cancer. 2006; 42:290-294. [PubMed: 16376541]

50. Dempsey EC, Newton AC, Mochly-Rosen D, Fields AP, et al. Protein kinase C isozymes and the regulation of diverse cell responses. Am J Physiol Lung Cell Mol Physiol. 2000; 279:L429-L438. [PubMed: 10956616]

51. Sabri A, Steinberg SF. Protein kinase C isoform-selective signals that lead to cardiac hypertrophy and the progression of heart failure. Mol Cell Biochem. 2003; 251:97-101. [PubMed: 14575310]

52. Kocinsky HS, Girardi ACC, Biemesderfer D, Nguyen T, et al. Use of phospho-specific antibodies to determine the phosphorylation of endogenous $\mathrm{Na}^{+} / \mathrm{H}^{+}$exchanger NHE3 at PKA consensus sites. Am J Physiol Renal Physiol. 2005; 289:F249-F258. [PubMed: 15687252] 

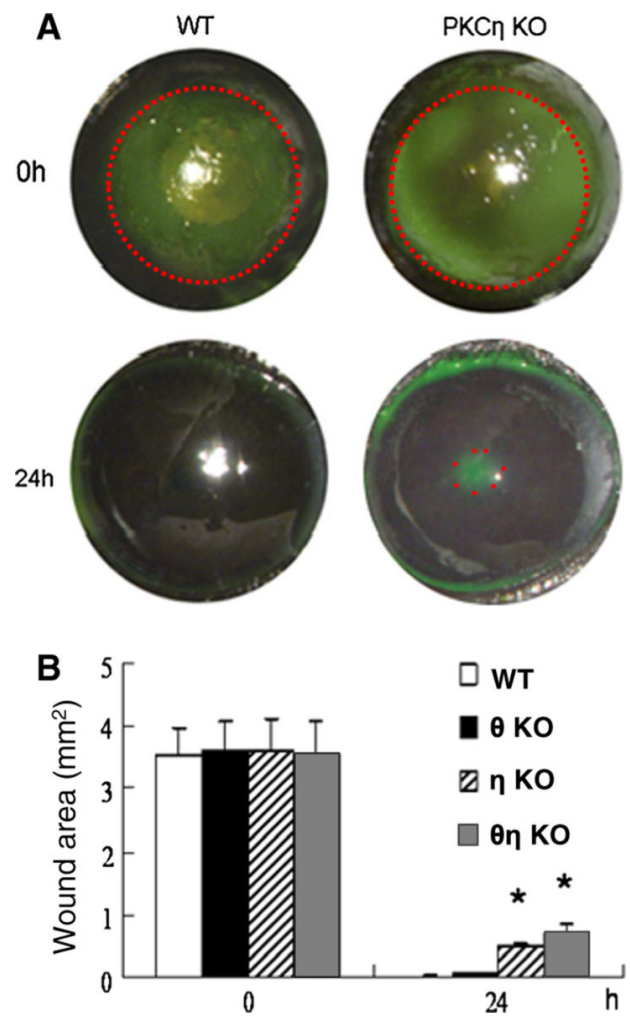

Fig. 1.

PKC knockout impaired corneal wound healing in a PKC $\eta$-specific manner. a Wounded cornea of wild-type and PKC $\eta$ knockout mouse. b Area measured at the wounded cornea of wild-type mouse and of PKC knockout [ $\eta$ (eta), $\theta$ (theta) and double; $\eta+\theta$ (eta + theta)] mouse $24 \mathrm{~h}$ after wounding. $n=6 . * p \leq 0.05$ 

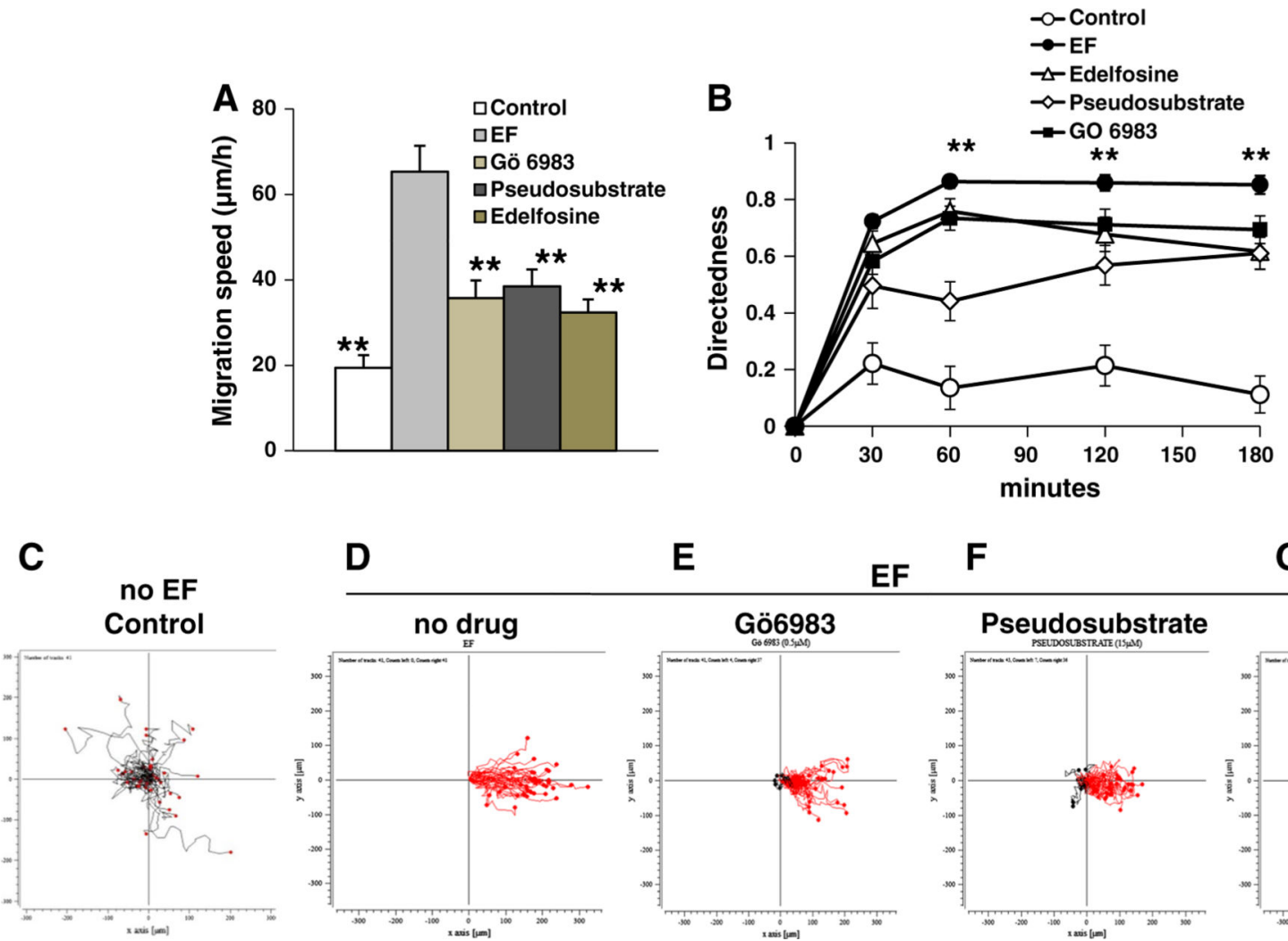

E

EF $F$
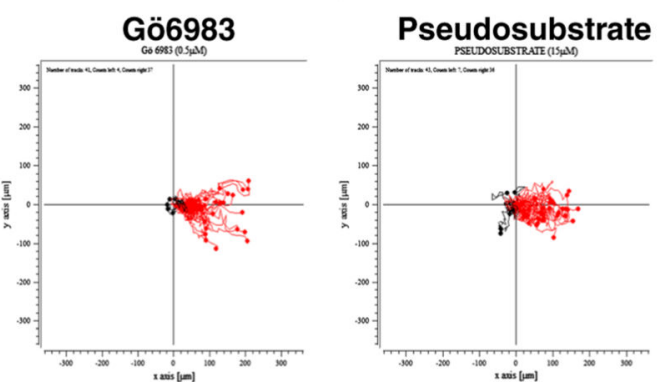

G

Fig. 2.

PKC inhibition reduced directional migration of HEK cells in EFs. a Displacement speed $(\mu \mathrm{m})$ and $\mathbf{b}$ directedness values of HEK cells $(\mathbf{c}-\mathbf{g})$ migration trajectories of cells. Control, EF, Gö6983 $(0.5 \mu \mathrm{M})$, pseudosubstrate $(15 \mu \mathrm{M})$, or edelfosine $(20 \mu \mathrm{M})$. Data are representative of three to four independent experiments (error bars, SEM). $* * * p \leq 0.001$ versus EF 

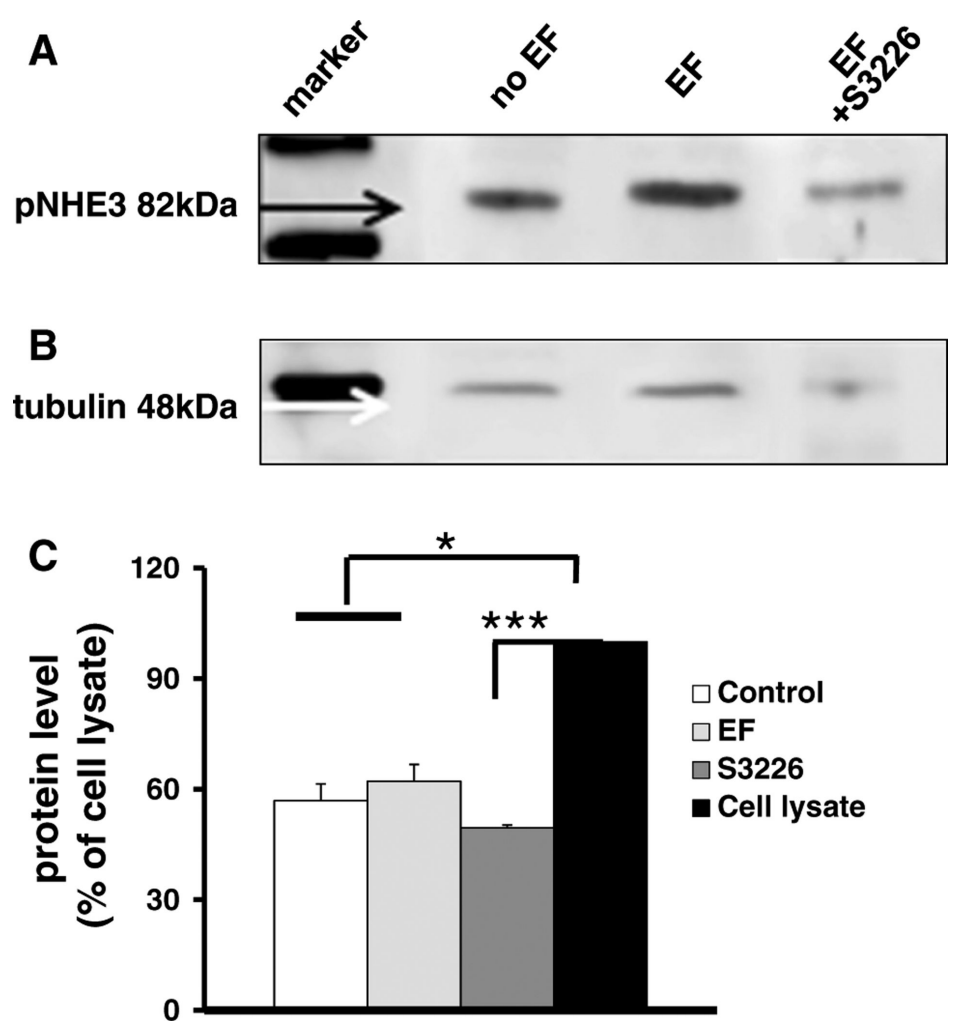

Fig. 3.

Pull-down assay showed complex formation of phospho-NHE3 with PKC $\eta$ and $\gamma$-tubulin. Immunoprecipitated cell lysates pulled down with and $\mathrm{PKC} \eta(\mathbf{a})$ and $\mathrm{pNHE} 3$ (b), respectively, showing interactions between pNHE3 and $\mathrm{PKC} \eta$ (a) pNHE3 and $\gamma$ tubulin (b). Upper row pulled down by PKCeta antibody, then detected in Western blot for pNHE3 (bands are shown by a black arrow). Lower row pulled down by $\mathrm{pNHE3}$ antibody, then detected in Western blot for tubulin (bands are shown by a white arrow). c Plot showing the percentage values of protein levels in immunoprecipitated control, EF, and EF + S3226 vs. control cell lysate. $n=3$. $* p \leq 0.05, * * * p \leq 0.001$ 
A

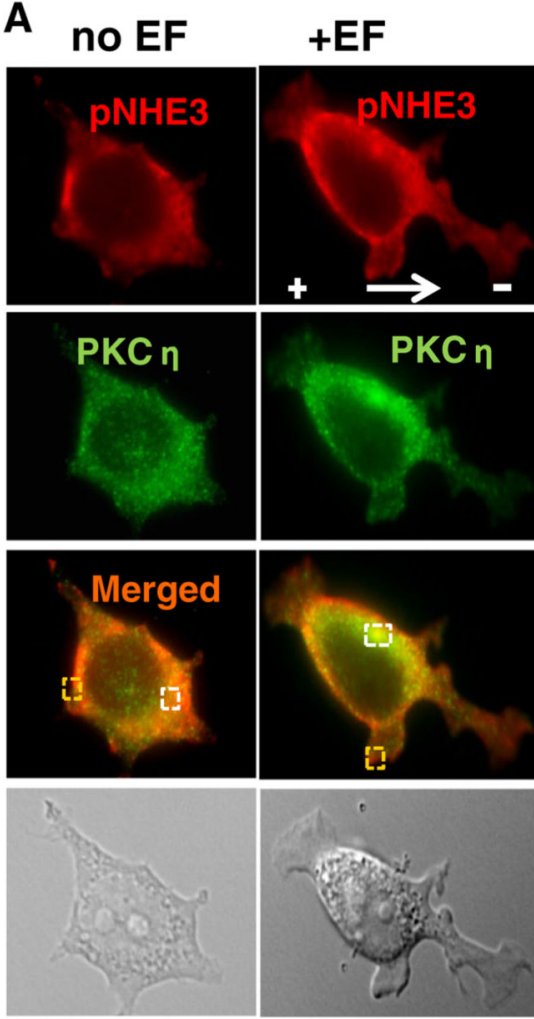

D

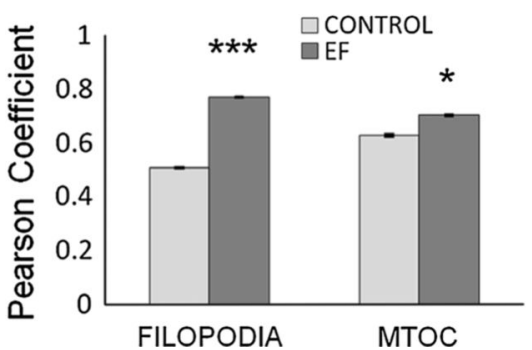

B
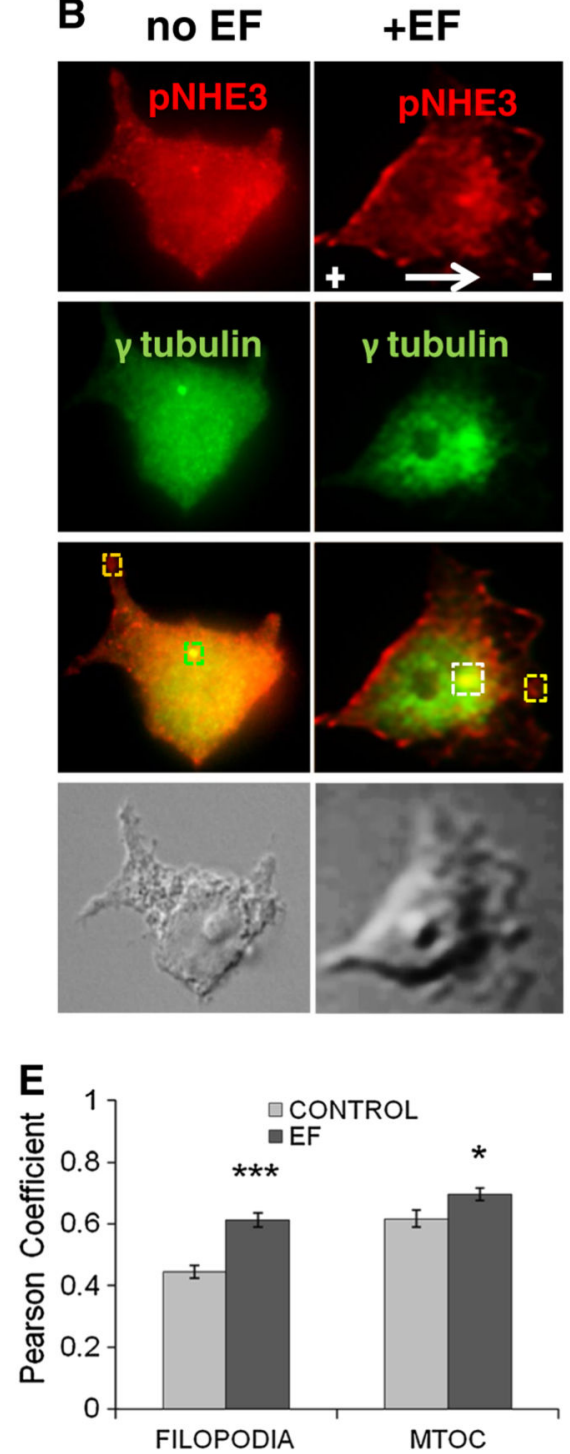
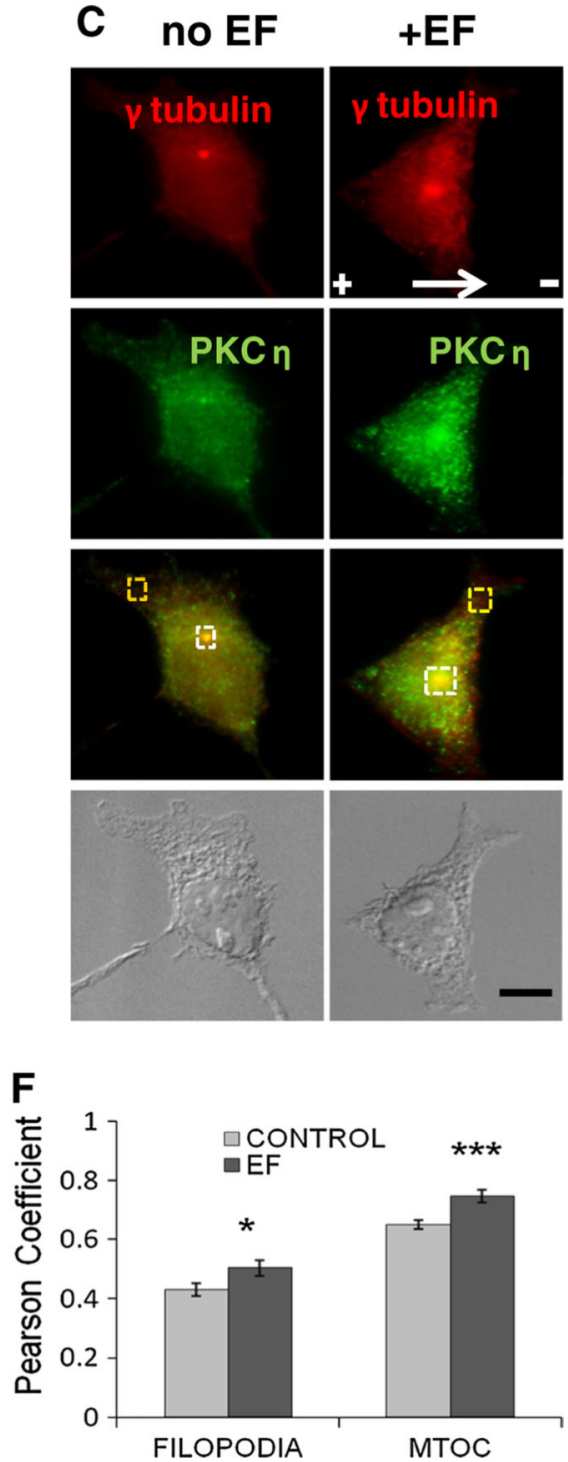

Fig. 4.

Colocalization of phospo-NHE3 with PKC $\eta$ and $\gamma$-tubulin. Fluorescence images of cells labeled against a pNHE3 and PKC $\eta, \mathbf{b}$ pNHE3 and $\gamma$ tubulin, and $\mathbf{c} \gamma$ tubulin and PKC $\eta$. pNHE3 and PKC appeared to have a biased distribution to leading edge and cell boundaries in migrating cells. Graphics showing colocalization rate between $\mathbf{d}$ pNHE3 and PKC $\eta \mathbf{e} \gamma$ tubulin and PKC $\eta$, and $\mathbf{f}$ pNHE3 and $\gamma$ tubulin at filopodia and at MTOC in control and EFexposed cells. Scale bar $10 \mu \mathrm{m}$. Data is representative of three independent experiments (error bars SEM). $* p \leq 0.05, * * * p \leq 0.001$ compared with control. $n=40$ cells 

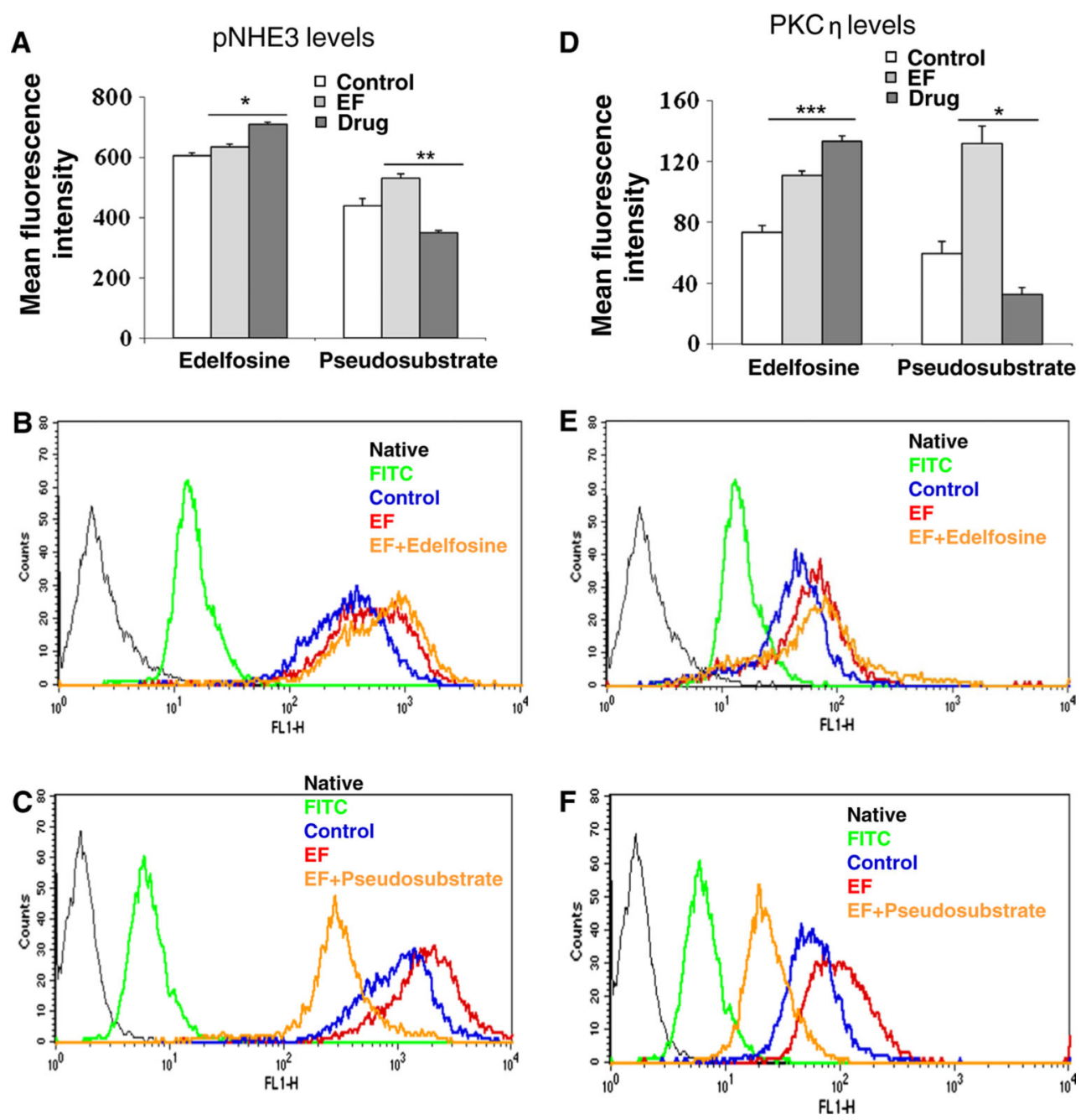

Fig. 5.

PI-PLC-independent activation of NHE3 via PKCๆ. FACS measurements representing intracellular levels of pNHE3. a-c and PKC $\eta \mathbf{d}-\mathbf{f}$ in control cells and in cells exposed to EF. Edelfosine and pseudosubstrate were used as PI-PLC and PKC $\eta$ inhibitors, respectively. Average cellular intensity of pNHE3.FITC (a) and PKC $\eta$.FITC (d) in control, EF and drug (either edelfosine or pseudosubstrate) conditions. Single histograms are representative examples from original FACS measurements showing fluorescence intensities of pNHE3.FITC and PKC .FITC scored from cells in control (no EF), EF and EF + drug conditions. In all measurements, native (unstained) and FITC (stained only with FITC) cells are included as internal control for the assay. ${ }^{*} p \leq 0.05, * * p \leq 0.01, * * * p \leq 0.001 . n=8$, each with 5,000 cells scored (error bars SEM) 

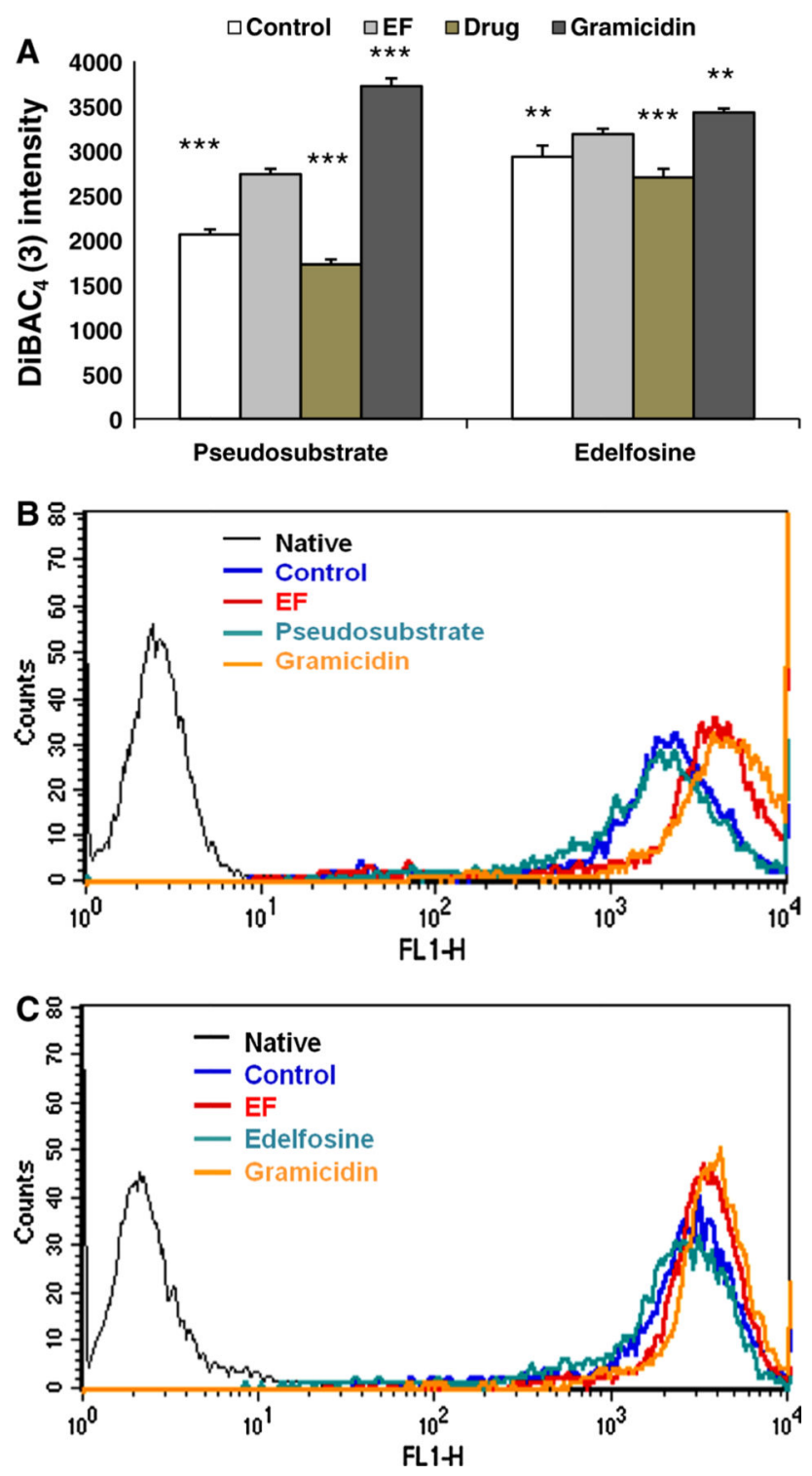

Fig. 6.

Activity of PKC $\eta$ regulates $V_{\text {mem }}$ changes. a-c FACS measurements representing membrane potential $\left(V_{\text {mem }}\right)$ of control cells and cells exposed to EF only, or combined with PI-PLC (edelfosine) or PKC $\eta$ inhibitors (pseudosubstrate). Cells were stained using voltage sensitive dye Bis-(1,3-Dibutylbarbituric Acid)Trimethine Oxonol ( $\left.\mathrm{DiBAC}_{4}(3)\right)$. Single histograms are representative examples from original FACS measurements showing fluorescence intensities of cells treated with pseudosubstrate (b) and edelfosine (c). In all measurements, native (unstained) and FITC (stained only with FITC) cells are included as internal control for the assay. Gramicidin is used as depolarization control. $* * p \leq 0.01$, ***p $\leq 0.001$. $n=8$, each with 5,000 cells scored (error bars SEM) 


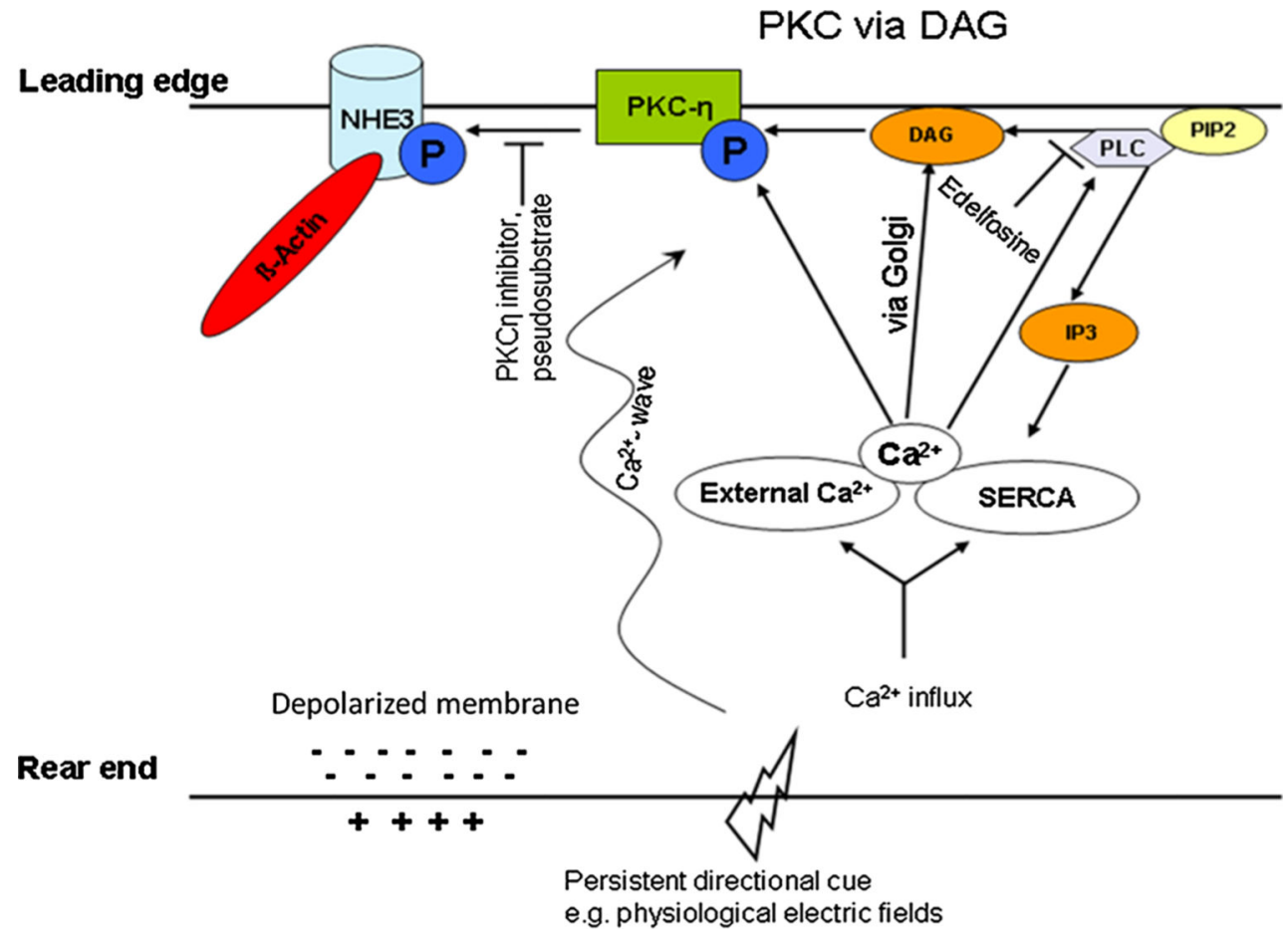

Fig. 7.

NHE3 activation via $\mathrm{PKC}$ in presence of directional cue (EF). Possible pathways that an $\mathrm{EF}$ can activate PKC, and then NHE3. Possible EF-downstream effectors are depicted from cell rear through the cytoplasm to the leading edge of a migrating cell 